\title{
Procesos de calidad en la educación militar. Reflexiones en torno a la formación en investigación en la Escuela de Armas Combinadas ${ }^{1}$
}

\author{
Juan Carlos Aristizábal Murillo \\ Escuela de Armas Combinadas del Ejército \\ Carlos Augusto Páez Murillo ${ }^{3}$ \\ Escuela Militar de Cadetes "General José María Córdova” \\ Andrés Mauricio Quintero Londoño ${ }^{4}$ \\ Escuela de Armas Combinadas del Ejército
}

\section{Resumen}

Objetivo: este trabajo hace una reflexión sobre los procesos de calidad en la educación de la Escuela de Armas Combinadas del Ejército Nacional (ESACE) a partir del análisis de las políticas, normatividades y planes que la institución ha aplicado con el ánimo de fortalecer su sistema educativo, así como del impacto que estas han tenido en los progresos investigativos de la comunidad. Materiales y métodos: este estudio, de tipo documental, construyó una matriz

1 Capítulo de libro que parte de los resultados del proyecto de investigación Práctica Pedagógica en el Ejército Nacional: Reflexiones en torno a la Formación y la Investigación, del Grupo de Investigación para la Capacitación Militar (GICAM) de la Escuela de Armas Combinadas (ESACE) registrado con el código COL0160714 de Minciencias. Los puntos de vista y los resultados de este capítulo pertenecen a los autores y no reflejan necesariamente los de las instituciones participantes.

2 Magíster en Educación con énfasis en Administración Educativa y Políticas Públicas de la Universidad Sergio Arboleda, y especialista en Docencia e Investigación Universitaria de la misma institución. Administrador de empresas de la Universidad Militar Nueva Granada y profesional en Ciencias Militares de la Escuela Militar de Cadetes. Orcid: https://orcid.org/0000-0002-3690-3282. Contacto: juanaristizabalmurillo@cedoc.edu.co

3 Doctor en Ingeniería de Sistemas del Instituto Politécnico Nacional (IPN) SEPI-ESIME Zacatenco. Cd. de México, México. Magíster en Ciencias en Ingeniería de Sistemas del IPN. Profesional en Ciencias Militares de la Escuela Militar de Cadetes. Orcid: https://orcid.org/0000-0002-5221-8437. Contacto: carlos.paez@ esmic.edu.co

4 Coronel del Ejército Nacional de Colombia. Profesional en ciencias militares de la Escuela Militar de Cadetes "General José María Córdova" y especialista en Seguridad y Defensa Nacional de la Escuela Superior de Guerra General Rafael Reyes Prieto. Coronel del Ejército Nacional de colombia; Magister en seguridad y defensa Nacional de Escuela de Guerra del Ejército; posgrado como director de seguridad en la universidad UDIMA de Madrid España; Especialización en administración de recursos militares y Especialización en docencia Universitariaen el Centro de Educación Militar CEMIL; profesional en ciencias Militares en la Escuela Militar de Cadetes José Maria Cordova; condecoración "Universitas Summa Cum Laude" por sobresalir en las obligaciones profesionales y las inherentes al estudio, la docencia, la investigación y la labor académica. Orcid: https://orcid.org/0000-0003-3422-9818. Contacto: andres.quinterolo@buzonejercito.mil.co 
de análisis de categorías a partir de una ecuación de búsqueda de palabras claves que permitió focalizar procesos de calidad en investigación para la ESACE. Resultados: el estudio evidencia avances en las líneas de investigación que coordinan y guían la producción académica, en cumplimiento al Plan Estratégico del Sistema Educativo (Pese). Conclusiones: se concluye que la Escuela ha propuesto soluciones o recomendaciones innovadoras, basadas en investigaciones de alto rigor técnico, tecnológico y científico, que involucran la transferencia de conocimiento según las necesidades o desafíos en la Fuerza.

Palabras clave: calidad de la educación; educación superior; Ejército Nacional de Colombia; investigación; normatividad.

\section{Introducción}

La educación es un proceso social que refleja las complejidades de las culturas, sus perspectivas de futuro y valores, así como la producción de conocimiento y las expresiones materiales y no materiales. Asimismo, genera sistemas de comprensión del mundo y concepciones, pues ofrece andamiajes que permiten al hombre transformar su medio, adaptarse y crear su narrativa. En Colombia se habla de educación con calidad después de la reforma estructural de la Constitución de 1991, que le permitió al Ministerio de Defensa diseñar e implementar políticas, planes y procesos que orientan a las comunidades académicas militares del país a lograr tales objetivos.

A partir de ese momento, la palabra calidad en la educación colombiana se ha transformado en una meta y acción de mejora en todas las instituciones académicas que forman parte del entramado educativo en el país. Esto incluye las áreas de formación de las Fuerzas Militares (FF. MM.), que han enfrentado la transición de los procesos académicos para acoplarlos a los criterios de calidad establecidos por la normatividad colombiana. Una evidencia de esta transformación fue el desarrollo del Proyecto Educativo de las Fuerzas Armadas (Pefa), el Sistema Educativo de las Fuerzas Armadas (Sefa) y el Plan Estratégico del Sistema Educativo de las Fuerzas Armadas (Pese), donde se adoptan lineamientos de calidad en la educación establecidos por la Organización de las Naciones Unidas para la Educación, la Ciencia y la Cultura (Unesco).

Una de estas instituciones fue la Escuela de Armas Combinadas (ESACE), la cual se encarga de capacitar y especializar a los oficiales y suboficiales del Ejército. Por lo tanto, la exigencia académica e investigativa es alta, ya que a partir de este centro se desarrollan ideas que permiten fortalecer el nivel educativo de la institución e innovar en las mismas técnicas que se emplean en diversos ámbitos del régimen militar.

En este sentido, el presente capítulo busca ofrecer al lector una serie de reflexiones sobre las políticas y procesos que se han desarrollado en torno a los 
estándares de calidad en la educación de la Escuela de Armas Combinadas. Para esto se analizan únicamente las directivas o normatividades generadas para mejorar los procesos de investigación o producción de literatura científica, ya que, como se mencionó, este ámbito es el encargado de gestionar el conocimiento e innovación en la Fuerzas Militares.

La ESACE ha sido parte de esta evolución y actualmente cuenta con el registro calificado de dos programas de posgrado que se lograron a través del Centro de Educación Militar (CEMIL), el cual ha desarrollado desde su creación prácticas pedagógicas alrededor de la investigación que han generado la mejora continua de los procesos y calidad de la educación en los oficiales subalternos que las realizan.

Estos argumentos conducen la siguiente reflexión: ¿De qué manera los procesos de calidad en la educación, específicamente en investigación, que ha realizado el Ejército Nacional contribuyen al mejoramiento continuo en la formación de los oficiales y suboficiales? Asimismo, surge esta pregunta específica: ¿Cómo se llevan a cabo los procesos de mejora de la calidad en la educación en la Escuela de Armas Combinadas del Ejército Nacional?

Con el fin de responder estas preguntas es necesario hacer una contextualización para comprender cómo ha sido el proceso de mejora continua que ha tenido el Ejército Nacional en educación, específicamente en investigación, y cómo esta ha impactado a los alumnos de la ESACE para fortalecer las capacidades de la institución.

\section{Marco teórico}

\section{Antecedentes}

Desde sus inicios y como institución que protege la seguridad del Estado, el Ejército Nacional se ha preocupado por el mejoramiento continuo en sus procesos educativos con el desarrollo de la política institucional de formación integral, gradual y permanente que prevé el desarrollo del potencial de sus integrantes y la formación integral que atienda el crecimiento personal y tecnológico en el campo militar.

Un hito en este proceso es la Carta Constitucional de Rionegro que el Estado colombiano firmó en 1863 y en la que quedó conformado por nueve estados federales, entre los que se encontraban Antioquia, Bolívar, Boyacá, Cauca, Cundinamarca, Magdalena, Panamá, Santander y Tolima (Kalmanovitz, 2013). Cada uno tenía autonomía y con capacidad de organizar sus propios ejércitos; es decir que en esa época existían nueve ejércitos en Colombia. Esta situación 
generó caos, ya que se presentaron conflictos entre los Estados porque cada uno quería establecer sus propias normas y leyes.

Por su parte, la nación tenía un ejército que se denominaba "Guardia Colombiana”. Aunque la misión principal de esta fuerza era intervenir en caso de que existiera un conflicto entre alguno de los ejércitos de los Estados, no era efectiva debido a que, por un lado, era pequeña y, por el otro, los Estados eran los que proveían de recursos humanos y financieros para dotarla. Por tal razón, si existía confrontación entre dos Estados, la Guardia Colombiana difícilmente podía obtener recursos para funcionar, de manera que su labor quedaba limitada para poder intervenir en esos conflictos.

Posteriormente, en 1886 se sancionó una nueva Constitución que centralizó los dos ámbitos de dominio indispensables de un Estado-nación: el monopolio fiscal y el de la fuerza. "De esa manera se dispuso que la nación debería tener para su defensa un ejército permanente unificado al servicio del poder regenerador" (Esteban, 2008, p. 153). Con esta decisión, se formalizó un solo ejército colombiano que permitió centralizar los recursos, las fuerzas y las capacidades para enfocarlos en una sola institución. Una vez unificada una fuerza para el país, los dirigentes se preocuparon por proporcionar una educación para sus oficiales y sus soldados. Al principio, los oficiales eran seleccionados de la tropa y la formación era orientada por sacerdotes de la iglesia Católica.

Más adelante, con el fin de unificar los criterios de educación para profesionalizar la fuerza, evitar el subjetivismo y aupar la meritocracia para seleccionar los oficiales, se creó la Escuela Militar de Cadetes, que funcionó de manera intermitente entre 1880 y 1907. En detalle, con el nombre de Escuela de Ingeniería Civil y Militar funcionó de 1880 a 1884, adscrita a la Secretaría de Guerra. Fue reabierta con la denominación Escuela Militar de Cadetes de 1889 a 1892, dependiente del Ministerio de Guerra, y reorganizada en 1891. De nuevo, "se reabrió durante 1898 y, sin otra interrupción, desde 1907” (Triana, 2011, p. 323).

Según la historia del Ejército Nacional de Colombia, para algunos autores 1907 es el año en el que comenzó la profesionalización de la fuerza. En sus inicios, la Escuela Militar fue dirigida por dos oficiales chilenos egresados de academias militares alemanas, quienes tenían la misión de organizarla y dirigirla; fundar cuerpos modelo como base para definir la estructura de la escuela de suboficiales; organizar cursos de aplicación para oficiales del Ejército que no habían pasado por ninguna escuela de formación; "elaborar e implementar los reglamentos orgánicos del Ejército y, finalmente, organizar el Alto Mando del Ejército, la Inspección General y el Estado Mayor General" (Leongómez, 1987, p. 23). 
Esta misión chilena duró poco tiempo debido a las "divergencias con el Ministerio de Guerra” (Helg, 1986, p. 4). Sin embargo, los presidentes de Colombia de la época querían seguir la reforma educativa del Ejército con el fin de tener una fuerza de calidad, pero ahora con asesoría de militares europeos. Sin embargo, debido a la Primera Guerra Mundial no tenían muchas opciones salvo Suiza, que no estaba muy comprometido con la guerra y tiene unas características de terreno montañoso similares a las de Colombia. Decidieron entonces tocar puertas e iniciar negociaciones hasta que en 1923 el gobierno del presidente Pedro Nel Ospina y el gobierno suizo firmaron un acuerdo que se prolongó hasta febrero de 1929. En términos educativos, esta alianza permitió al Ejército alcanzar lo siguiente (Helg, 1986):

1. En marzo de 1925 se abre en Bogotá la Escuela de Cadetes y Suboficiales, plantel destinado a la formación de los cabos y sargentos encargados del mando y de la instrucción de los conscriptos.

2. La Escuela Militar de Cadetes se transforma con la gestión del mayor Hans Von Werdt: pasa del colegio de enseñanza general a un establecimiento militar especializado, al cual no podrían ingresar sino jóvenes de 16 años de edad con vocación para la carrera de las armas, pues entraban a la escuela a hacer el bachillerato, pero cuando lo obtenían, la gran mayoría desertaba porque no quería seguir la carrera militar.

3. Concentrando sus intervenciones en la instrucción militar y la elaboración de proyectos sectoriales, la misión no puede transformar los fundamentos del Ejército colombiano: no le quita su papel de agente de represión al servicio del Partido Conservador, de la oligarquía y de las compañías extranjeras para volverlo una fuerza nacional de defensa territorial.

4. Formó una nueva generación de oficiales competentes y concienzudos, los cuales exigirían después reformas y responsabilidades a la altura de su capacitación.

5. Inicia la profesionalización de los oficiales. Como otros puestos en la administración pública, a partir de 1925 la oficialidad deja de ser un privilegio de las clases altas para volverse un canal de ascenso social para la clase media. A pesar de que no duró mucho la influencia de la comisión suiza debido a diferencias con militares colombianos, en algo contribuyeron a mejorar los procesos de calidad de la educación del Ejército: se debió pensar en crear una escuela diferente para suboficiales que contribuyeran con el mando de las tropas. 
Luego de este período, el Ejército fue influido por una misión alemana, que entre varias de sus labores lo organizó territorialmente para tratar de garantizar el monopolio del control territorial del país. Así, asignó cinco brigadas, las cuales se establecieron de la siguiente manera: la primera brigada tendría su sede en Bogotá, la segunda en Barranquilla, la tercera en Cali, la cuarta en Medellín y la quinta en Bucaramanga (González, 2009). Esta misión estaba en Colombia principalmente por la cercanía que tenían con Chile; asimismo, contribuyeron significativamente en el conflicto que Colombia tuvo con el Perú.

Al terminar la Segunda Guerra Mundial y después de que Colombia participara en la guerra de Corea entre 1951 y 1954 , "se asimila la doctrina militar americana por medio de la apropiación de la técnica y la táctica de guerra” (Buitrago, 2006, p. 123). Paralelo a estos acontecimientos, bajo el Decreto 2488 del 6 de octubre de 1936 se crea la Escuela de Aplicación de Caballería, el 1. ${ }^{\circ}$ de noviembre de 1936 (Ejército Nacional, s. f). Esta escuela se levantó donde se asentaba el Grupo de Caballería N. 1 (Páez), en la hacienda Santa Ana, por disposición del gobierno de Bogotá de la época. La construcción de una carretera divide en dos esa hacienda y, en uno de sus lados, se constituye la Escuela de Infantería. Ambas escuelas dieron origen a la Brigada de Institutos Militares.

Posteriormente La Brigada de Institutos Militares pasó allamarse Decimoquinta Brigada (Centro de Instrucción Militar, 2017), creada mediante la Disposición 015 del 18 de diciembre de 1995, que funcionó en las instalaciones del Cantón Norte, con la dirección del coronel Eduardo Antonio Herrera Verbel, hasta que en el año de 1999 pasó a ser el Centro de Educación Militar (CEMIL) (Ejército Nacional, 2017, p. 8). Este cambio ajustó el nuevo contexto educativo adoptado por la institución, el cual consistió en separar la instrucción del entrenamiento y de esta manera dio paso a la creación del Centro Nacional de Entrenamiento (Cenae) (Centro de Instrucción Militar, 2017).

Posteriormente, mediante la Disposición N. ${ }^{\circ} 0007$ del 23 de junio de 1980, se creó el Instituto de las Armas y los Servicios (IAS), conformado por la Inspección de Estudios de las Escuelas de las diferentes especialidades y con la misión específica de desarrollar cursos para ascensos de oficiales y suboficiales de las armas y los servicios. Es precisamente allí donde nace la Escuela de Armas Combinadas del Ejército (ESACE). En esta contextualización se evidencia la manera como el Ejército ha venido evolucionando y con él los procesos educativos, particularmente en la Escuela de Armas, que es el objeto de estudio de esta reflexión.

Años después, el artículo 222 del capítulo 7 de la Constitución de 1991 decretó que "la ley determinará los sistemas de promoción profesional, cultural y social de los miembros de la Fuerza Pública. En las etapas de su formación, se 
les impartirá la enseńanza de los fundamentos de la democracia y de los derechos humano" (República de Colombia, 1991, p. 113). El Ejército no ha estado transformando estos procesos solo, sino que también es producto de la tendencia que ha tenido el país por mejorar sus procesos de educación. Al respecto, el artículo 67 de la Constitución Política de 1991 dice que

la educación es un derecho de la persona y un servicio público que tiene una función social; con ella se busca el acceso al conocimiento, a la ciencia, a la técnica, y a los demás bienes y valores de la cultura. (República de Colombia, 1991)

A partir de esta Constitución, el país ha implementado estrategias y herramientas para alcanzar estándares de calidad que permitan mejorar la educación y la capacitación de los estudiantes, así como impactar en el cumplimiento de la misión constitucional para construir sistemas socialmente robustos coordinados e integrados en beneficio de la sociedad (Páez, Peón \& Ramírez, 2018).

\section{Calidad en la educación superior en Colombia}

En Colombia las temáticas referentes a la educación empezaron a tomar relevancia desde los años 60, lo cual concuerda temporalmente con las conferencias sobre educación que ofreció la Unesco en diferentes países de Latinoamérica, como Argentina y Venezuela, donde se abordaron temas como la capacitación del docente y el uso de tecnologías en los procesos de educación. Alternamente en el país, se hablaba sobre la expansión de la educación primaria en las zonas rurales.

Posteriormente, en los años 90 el país intentó ajustar sus lineamientos de políticas educativas con los planteamientos expuestos en las conferencias dirigidas por la Unesco, para lo cual expidió normativas como la Ley 30, de diciembre 28 de 1992, por la cual se organiza el servicio público de la Educación Superior y cuyo objetivo fundamental es "garantizar a la sociedad que las instituciones que forman parte del sistema cumplan los más altos requisitos de calidad y que realizan sus propósitos y objetivos" (República de Colombia, 1992). Si bien las universidades hacen voluntariamente esta acreditación, el Estado otorga beneficios a las instituciones que la realizan y la sociedad las reconoce por ser entidades acreditadas en alta calidad. Cabe anotar que dicha acreditación se otorga por un periodo determinado.

Consecuentemente, en 1992 se establecieron estamentos de inspección y vigilancia a cargo del Consejo Nacional de Educación Superior (Cesu) y por el Instituto Colombiano para el Fomento de la Educación Superior (Icfes) $)^{5}$, los cuales

5 Actualmente el nombre de esta entidad es Instituto Colombiano para la Evaluación de la Educación. 
fueron encargados de garantizar y vigilar la calidad de la educación en Colombia, entre muchas otras funciones.

A su vez, con la Ley 115 de febrero 8 de 1994 se decretó la Ley General de Educación, con la cual se cambia la visión educativa y el plan decenal de educación proyectado desde 2016 al 2026, los cuales formulaban que la calidad en la educación debía promocionar la participación autónoma en los procesos de acreditación educativa e investigación para gestionar el conocimiento.

De esta manera, el Consejo Nacional de Acreditación en 1998 y el Ministerio de Educación de Colombia en 2000 empezaron a resaltar la importancia de inculcar en la educación la identidad, la autonomía, la historia y los hábitos investigativos. Con este espíritu se desarrolló el proyecto de reestructuración y acreditación de la educación superior, el cual tuvo que solucionar los problemas de conceptualización del concepto de calidad en la educación para estandarizar criterios y factores de desarrollo en este ámbito. Para ello se analizaron las problemáticas que sufre la educación y se obtuvo como resultado la formulación de metas que atacarían desde las entrañas las problemáticas educativas.

El primer aspecto mencionado fue la función de la educación en la sociedad. Para Jaramillo (2004), el país debe formar mano de obra capaz de enfrentar los criterios de calidad implementados en la ejecución de procesos en las empresas. Dado que estos estándares provienen del exterior debido a los flujos de la globalización, para asumir una posición competitiva frente al mercado global deben adaptarse a dichos cambios, los cuales solo pueden ser asumidos con una formación y educación estructurada con esos lineamientos (Jaramillo, 2004).

El segundo aspecto expuesto fue la humanización de la sociedad. Al respecto, Jaramillo (2004) enfatiza en la necesidad de formar profesionales éticos, es decir, propone una educación fundamentada en principios y valores que permitan una convivencia ciudadana sana irradiada por sentimientos o deseos de superación personal.

El tercer aspecto que se resaltó en este proceso fue el concepto de identidad. Magendzo (1986), citado por Jaramillo (2004), explica que una educación con calidad debe formar a una comunidad que esté en la capacidad de darle significancia y contexto a su cotidianidad. Es decir, que tenga las condiciones de reconocer la situación de su país y aceptarla o cambiarla, lo cual hace que en la sociedad brote un sentir de pertenencia y relación con el entorno que genera estados de progreso y bienestar. Finalmente, como cuarto aspecto este autor abordaba la investigación. Particularmente, menciona que en las memorias del Congreso Pedagógico Nacional de 1987 se consideró la educación con calidad como aquella que despierta 
el gusto por el conocimiento y que, por lo tanto, fomenta la escritura científica, la investigación y la innovación (Jaramillo, 2004).

Como se puede observar, la educación en Colombia no ha sido ajena a esta tendencia mundial, en la cual se considera que la calidad de la educación superior consiste en las características que permiten reconocer un programa académico específico o una institución de determinado tipo y hacer un juicio sobre la distancia relativa entre el modo como en esa institución o en ese programa académico se presta dicho servicio que corresponde a su naturaleza (Consejo Nacional de Acreditación, 2013, p. 12). Es decir, la calidad garantiza que la institución de educación superior está prestando un excelente servicio a la comunidad a la cual se debe.

En el año 2000, "el Consejo Nacional de Acreditación elaboró los Lineamientos para la Acreditación Institucional” (Asociación Colombiana de Universidades, 2006). También en ese año se creó la Comisión Nacional Intersectorial para el Aseguramiento de la Calidad (Conaces) y se fortaleció el Consejo Nacional de Acreditación (CNA). Además, se creó un conjunto de sistemas de información con el fin de contribuir al conocimiento y la toma de decisiones del sector, dentro de los cuales se encuentran el Sistema Nacional de la Información de la Educación Superior (Snies), el Sistema de Información para el Aseguramiento de la Calidad de la Educación Superior (Saces), el Sistema para la Prevención de la Deserción en Educación Superior (Spadies) y el Observatorio Laboral para la Educación (Melo, Ramos \& Hernández, 2014, p. 9).

Durante los primeros años del siglo XXI la educación en el país ha transitado por buenos caminos. Según la Organización para la Cooperación y el Desarrollo Económicos (OCDE y Banco Mundial, 2012), se ha aumentado la cobertura, ha variado el panorama institucional, el gobierno nacional ha planificado y formulado con coherencia y solidez políticas en temas de educación, existe un fuerte apoyo a la equidad y el sistema de créditos educativos es uno de los mejores de la región. Asimismo, para asegurar los estándares de calidad, las instituciones de educación superior en Colombia son evaluadas en dos momentos: en el primero, de carácter obligatorio, se verifican quince condiciones mínimas de calidad para que dicho programa o institución se pueda ofertar al público. El otro momento es voluntario y es cuando se realiza la acreditación institucional o de alta calidad. La tabla 1 muestra la comparación de los decretos 1075 de 2015, 1280 de 2018 y 1330 de 2019 en cuanto a las exigencias que se hace a las IES respecto a la investigación. Estos decretos reglamentan todo el proceso de registro calificado. 
Tabla 1. Exigencias en investigación a las Instituciones de Educación Superior

\section{Decreto 1075 del 26 de mayo de 2015}

Investigación. Actividades de investigación que permitan desarrollar una actitud crítica y una capacidad creativa para encontrar alternativas para el avance de la ciencia, la tecnología, las artes o las humanidades y del país, de acuerdo con las orientaciones que se indican a continuación.

- El programa debe prever la manera como va a promover la formación investigativa de estudiantes o los procesos de investigación, o creación, en concordancia con el nivel de formación y sus objetivos.

- El programa debe describir los procedimientos para incorporar el uso de Tecnologías de la Información y la Comunicación en la formación investigativa de los estudiantes.

Para la adecuada formación de los estudiantes se verificará:

- La existencia de un ambiente de investigación, innovación o creación, el cual exige políticas institucionales en la materia; una organización del trabajo investigativo que incluya estrategias para incorporar los resultados de la investigación al quehacer formativo y medios para difundir los resultados de investigación. Para los programas nuevos de pregrado el proyecto previsto para el logro del ambiente de investigación y su desarrollo, que contenga por lo menos recursos asignados, cronograma y los resultados esperados.

- Los productos de investigación en los programas en funcionamiento de pregrado y posgrado y los resultados de investigación con auspicio institucional, para los programas nuevos de maestría y doctorado.

- Los resultados de procesos creativos de los programas en artes, podrán evidenciarse en exposiciones, escenificaciones, composiciones o interpretaciones y sustentarse en registro de la obra, estudios el campo artístico y publicaciones en diversos formatos.

- En los programas de maestría y doctorado la participación de los estudiantes en los grupos de investigación o en unidades de investigación del programa.

- La disponibilidad de profesores que tengan a su cargo fomentar la investigación y que cuenten con asignación horaria destinada a investigar títulos de maestría o doctorado o experiencia y trayectoria en investigación demostrada con resultados debidamente publicados, patentados o registrados.

\section{Decreto 1280 del 25 de julio de 2018}

Desde el punto de vista institucional y con el propósito de garantizar la cultura de formación en investigación, esta condición valora que la institución cuente con una política de fomento de la investigación, desarrollo, innovación, creación artística, caracterización y generación de nuevos productos, y formación de los estudiantes en actitud crítica y responsable, y que, a su vez, cuente con los recursos para hacer la gestión viable. Cuando se trate de instituciones que se declaran con énfasis o enfoque en ser instituciones de educación superior de investigación, y/o de instituciones que entre su oferta académica cuenten con programas de maestría en investigación y doctorado, en esta condición se valorarán los desarrollos que sustentan dicha declaración y oferta.

Investigación. Para los programas de pregrado, especialización y maestrías de profundización, se evaluará la formación básica en investigación en la condición de gestión curricular. Para el caso de los programas de maestría de investigación y doctorado, esta condición valora los desarrollos que sustentan dicha formación y, teniendo en cuenta la naturaleza del programa, también se valora la creación de conocimiento la creación artística o innovación, así: 


\section{Decreto 1280 del 25 de julio de 2018}

La creación de conocimiento expresa el proceso de investigación propiamente dicho, que implica alizar el conocimiento existente, someterlo a prueba de nuevas hipótesis y concluir con el descubrimiento o generación de nuevo conocimiento.

La creación artística se asume como producción que se lleva a cabo a través de una exposición, pieza musical, obra de teatro, o proyecto de diseño que se realiza desde las artes y no desde su observación, y bajo perspectivas disciplinares diversas que se basan en las prácticas artísticas, y cuyos procesos, estructura y resultados son transferibles y replicables a centros investigadores.

La innovación puede ser vista como la transformación de una idea, el mejoramiento de un producto o servicio. El desarrollo de nuevos procedimientos o métodos donde se logre como característica esencial agregar valor científico, productivo o de capacidades.

Parágrafo 1. El Ministerio de Educación Nacional definirá los instrumentos y lineamientos necesarios para el desarrollo del proceso de evaluación de las condiciones de programa.

Parágrafo 2. Las instituciones deberán presentar la información necesaria y suficiente que permita verificar la manera en que se implementan las políticas de educación inclusiva en cada una de las condiciones de calidad previstas en este artículo, de acuerdo con los lineamientos que el Ministerio de Educación Nacional establezca para ello y con las disposiciones legales que así lo exigen.

\section{Decreto 1330 del 25 de julio de 2019}

Investigación, innovación y/o creación artística y cultural. La institución deberá establecer en el programa las estrategias para la formación en investigación-creación que les permitan a profesores y estudiantes estar en contacto con los desarrollos disciplinarios e interdisciplinarios, la creación artística, avances tecnológicos y el campo disciplinar más actualizado, de tal forma que se desarrolle el pensamiento crítico y/o creativo.

El programa, en coherencia con el nivel de formación, las modalidades (presencial, a distancia, virtual, dual u otros desarrollos que combinen e integren anteriores modalidades), con la naturaleza jurídica, tipología, identidad y misión institucional, propenderá a que sus resultados de investigación contribuyan a la transformación social de las dinámicas que aporten a la construcción del país.

Según la declaración explícita que realice el programa en relación con la incorporación de la investigación para el desarrollo del conocimiento, el programa deberá definir las áreas, líneas o temáticas de investigación en las que se enfocarán los esfuerzos y proyectos. Lo anterior, teniendo en cuenta los siguientes propósitos de investigación:

a) La comprensión teórica para la formación de un pensamiento innovador, con capacidad de construir, ejecutar, controlar y operar los medios y procesos para la solución de problemas que demandan los sectores productivos y de servicios del país.

b) La incorporación de la formación investigativa de los estudiantes en concordancia con el nivel educativo y sus objetivos, el uso de las tecnologías de la información y de la comunicación.

c) El desarrollo de nuevos productos, procesos y usos de productos ya existentes.

d) La capacidad para dar soluciones transformadoras a problemas locales, regionales y globales, e indagar sobre la realidad social y ambiental, entre otros, a partir del uso del conocimiento como herramienta de desarrollo.

e) Aquellos programas que hicieron explícita la incorporación de la investigación, innovación y/o creación artística deberán evidenciar sus resultados de acuerdo con los lineamientos establecidos por el sistema nacional de ciencia y tecnología u otros afines.

Fuente: Original de los autores con información de los decretos mencionados (Ministerio de Educación Nacional [MEN], 2015, 2018, 2019). 
De esta manera, se puede apreciar que los criterios de calidad en Colombia han avanzado desde que en el año 2015 se propusieron las primeras reglamentaciones específicas referentes al proceso de investigación académica. Además, en vista de que todos los procesos son cambiantes, en el año 2018 se actualizó la normatividad el Decreto 1280 con el fin de garantizar la cultura de la investigación educativa, y en 2019 se publicó el Decreto 1330. Por supuesto, las Fuerzas Militares no han sido ajenas a esas exigencias y cada día se están actualizando con el fin de ajustarse a la ley y cumplir con la formación de los oficiales y suboficiales que la integran.

\section{Sistema Educativo de las Fuerzas Armadas (Sefa)}

La globalización es un proceso que ha impactado cada rincón del mundo en las diferentes dimensiones que lo componen: política, económica, social, tecnológica y, efectivamente, educativa. Ante esta hazaña de evolución que enfrenta la humanidad, se hizo necesario reestructurar los procesos educativos y de aprendizaje, ya que las habilidades de las personas también cambiaron.

De esta manera, en La educación esconde un tesoro la Unesco (1996) estableció una serie de principios enfocados en la educación a largo plazo y definió cuatro pilares que fundamentan dicho requerimiento. En este punto la pregunta que surge es si las fuerzas militares atenderán semejantes exigencias en sus normatividades educativas. Y la respuesta es que sí. El Ministerio de Defensa colombiano, teniendo en cuenta su misión y visión de un país democrático caracterizado por su progreso nacional, se apoyó en los cuatro pilares que definió la Unesco: (1) aprender a conocer, (2) aprender a hacer, (3) aprender a vivir juntos y (4) aprender a ser (Unesco, 1996, p. 34).

A partir de este referente se desarrolló el Sistemas Educativo de las Fuerzas Armadas, el cual se define "como conjunto de elementos interrelacionados en un círculo virtuoso para formar, actualizar, capacitar, instruir y entrenar a los integrantes de las Fuerzas Armadas" (Ministerio de Defensa Nacional, 2008). Así planteado, el Sefa garantiza un aprendizaje íntegro que fortalece cada una de las habilidades que exige la revolución tecnológica que atañe al mundo.

Específicamente, este sistema está apoyado en cuatro subsistemas que buscan fortalecer el activo vital de las Fuerzas Armadas: el recurso humano (figura 1).

La figura 1 muestra que los subsistemas fueron diseñados conforme a la visión del militar y policía del siglo XXI, los cuales facilitan el desarrollo del sistema:

- Gestión de doctrina: apoya lo procesos de investigación y análisis de la doctrina de las FF. MM. dentro de un marco legal (Ministerio de Defensa Nacional, 2008, p. 15). 
- Aseguramiento de la calidad: evalúa y asegura la empleabilidad, estándares y características de calidad en cada uno de los procesos educativos de la institución (Ministerio de Defensa Nacional, 2008, p. 15).

- Certificación militar y policial: hace referencia a los programas de formación, actualización, capacitación, instrucción y entrenamiento que tienen el objetivo de fortalecer las competencias individuales y colectivas que componen a un líder (Ministerio de Defensa Nacional, 2008, p. 15).

- $\quad I+D+I$ : soluciones o recomendaciones innovadoras, basadas en investigación de alto rigor técnico, tecnológico y científico, que involucren la transferencia de conocimiento, problemas, necesidades o desafíos en cada fuerza (Ministerio de Defensa Nacional, 2008, p. 16).

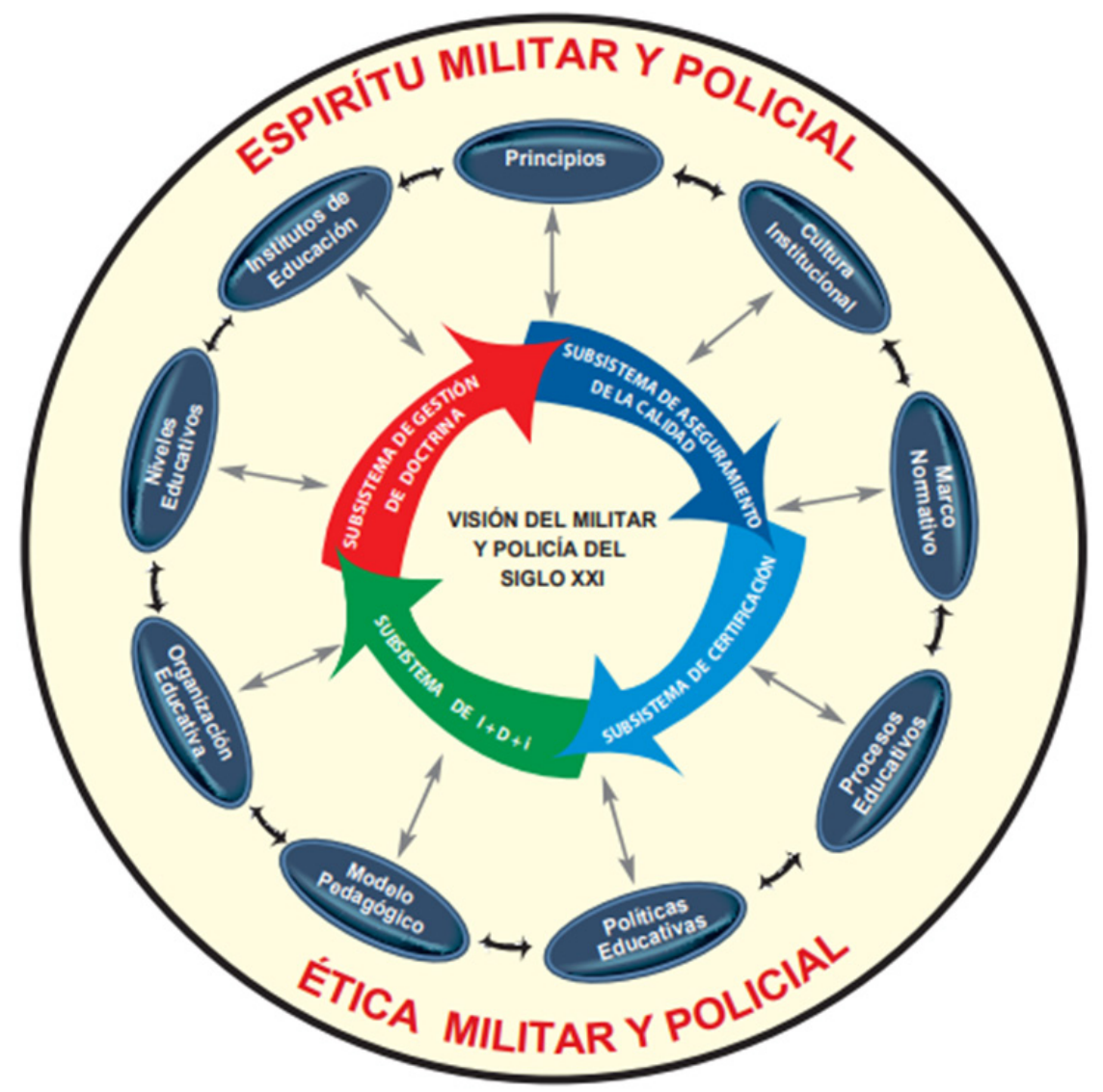

Figura 1. Estructura del Sistema Educativo de las Fuerzas Armadas (Sefa). Fuente: Ministerio de Defensa Nacional (2008). 
Cabe señalar que estos mecanismos facilitan el uso y el cumplimiento de las trece políticas educativas, las cuales buscan fortalecer cada uno de los pilares establecidos y fomentados por la Unesco (tabla 2).

Tabla 2. Políticas educativas del Sistema Educativo de las Fuerzas Armadas

\begin{tabular}{|c|c|}
\hline Política educativa & Descripción \\
\hline $\begin{array}{l}\text { Reforzar la formación en las } \\
\text { "competencias del ser" }\end{array}$ & $\begin{array}{l}\text { Formación de principios, valores, virtudes, ética } \\
\text { profesional y criterio militar y policial }\end{array}$ \\
\hline $\begin{array}{l}\text { Potenciar la educación profesional } \\
\text { militar y policial }\end{array}$ & $\begin{array}{l}\text { Requisito para ascenso de los oficiales y suboficiales de las } \\
\text { Fuerzas Militares }\end{array}$ \\
\hline $\begin{array}{l}\text { Transformar a la institución armada } \\
\text { en una "organización de aprendizaje" }\end{array}$ & Orientación hacia la investigación y la innovación \\
\hline $\begin{array}{l}\text { Educar de manera ininterrumpida } \\
\text { y ascendente }\end{array}$ & $\begin{array}{l}\text { Especialización y capacitación durante toda la carrera } \\
\text { militar }\end{array}$ \\
\hline $\begin{array}{l}\text { Redimensionar los currículos para } \\
\text { fundamentarlos en competencias }\end{array}$ & $\begin{array}{l}\text { Actualización del currículo reorganizado en pro de las } \\
\text { competencias del profesional militar }\end{array}$ \\
\hline $\begin{array}{l}\text { Establecer un modelo educativo } \\
\text { institucional de alta calidad }\end{array}$ & $\begin{array}{l}\text { Se encuentra conformado por la gestión del aseguramiento } \\
\text { y la acreditación de la calidad }\end{array}$ \\
\hline $\begin{array}{l}\text { Implementar la formación por ciclos } \\
\text { propedéuticos }\end{array}$ & $\begin{array}{l}\text { Secuencia complementaria de la educación del profesional } \\
\text { militar donde se evidencia la acumulación de créditos } \\
\text { académicos para cada nivel académico }\end{array}$ \\
\hline $\begin{array}{l}\text { Promover la internacionalización } \\
\text { educativa }\end{array}$ & Intercambios educativos \\
\hline $\begin{array}{l}\text { Aprender una o más lenguas } \\
\text { extranjeras }\end{array}$ & $\begin{array}{l}\text { Aprendizaje de una segunda lengua para facilitar la } \\
\text { internacionalización educativa }\end{array}$ \\
\hline Enfoque inter- $y$ transdisciplinario & $\begin{array}{l}\text { Nuevas formas de aprender y analizar los problemas del } \\
\text { contexto político, social y educativo }\end{array}$ \\
\hline $\begin{array}{l}\text { Privilegiar el autoaprendizaje } \\
\text { significativo }\end{array}$ & $\begin{array}{l}\text { Fortalece la memorización comprensiva para gestionar } \\
\text { nuevos conocimientos }\end{array}$ \\
\hline $\begin{array}{l}\text { Fortalecer la formación para } \\
\text { el liderazgo militar y policial }\end{array}$ & $\begin{array}{l}\text { Columna vertebral de la formación profesional militar } \\
\text { y policial }\end{array}$ \\
\hline
\end{tabular}

Fuente: Original de los autores.

Teniendo en cuenta estas políticas, las instituciones educativas pertenecientes a las FF. MM. reestructuraron su pensum, ya que las materias se debían amoldar al fortalecimiento de cada una de las habilidades que potencian las políticas del sistema y, evidentemente, la educación a largo plazo. 
De esta manera se da cuenta del compromiso que tiene la institución militar con los retos que pone el mundo antes sus pies. Es decir, los enfoques establecidos se orientan hacia una continua mejora que busca educar hombres y mujeres líderes íntegros con un sentir social y un criterio científico que permite obtener resultados óptimos.

\section{Escuela de Armas Combinadas (ESACE)}

Como se había mencionado anteriormente, la Esace es una escuela perteneciente al Centro de Educación Militar de las Fuerzas Militares de Colombia que busca capacitar y especializar a los oficiales y suboficiales del Ejército Nacional. Por lo tanto, teniendo en cuenta el Sistema de Educación establecido por el Ministerio de Defensa, la institución implementó el Proyecto Educativo de las Fuerzas Armadas con el objetivo de cumplir con los requisitos de calidad para la educación superior. Actualmente cuenta con dos programas de posgrado: la Especialización en Conducción y Administración de Unidades Militares (Snies 53894), para los oficiales que ascienden de tenientes a capitanes, y la Especialización en Administración de Recursos Militares para la Defensa Nacional (Snies 54014), para los oficiales que ascienden al grado de capitán a mayor. Además, ofrece siete cursos cortos (seis meses) con la finalidad de mejorar las capacidades operativas y de mando del profesional militar (Esace, 2019a, 2019b).

Estos programas nacieron debido a la reestructuración del Sistema Educativo de las Fuerzas Armadas. Esto significa que la Esace adecuó sus programas teniendo en cuenta el Proyecto Educativo de las fuerzas Armadas, en el cual se afirma que

una institución armada es mucho más que armas y municiones, mucho más que uniformes y combates, y mucho más que disciplina y reglas. Una institución armada es, ante todo, los hombres y mujeres que la componen, y de sus calidades humanas y profesionales deriva su éxito o fracaso. Por ello tienen tanta trascendencia los avances que se logren en el campo de la educación y la formación de sus integrantes. (Ministerio de Defensa Nacional, 2008, p.3)

Este proceso de transformación derivó en la formulación del Plan Estratégico del Sistema Educativo de las Fuerzas Armadas 2007-2019, el cual fue explicado en apartados anteriores. En este contexto, la Esace transformó su organización como institución de enseñanza, destacada por su labor de investigación científica y tecnológica, con base en un análisis juicioso de la doctrina que orientó la reestructuración de la educación de las Fuerzas Militares. En concordancia con estos principios, los estudiantes de la institución se ejercitan en el arte de la duda mediante el desarrollo del pensamiento complejo para alcanzar un sólido criterio militar. 
Asimismo, se debe mencionar que la política de calidad para la educación que se estableció para las Fuerzas Militares creó un macroproceso denominado Gestión Educación y Doctrina, compuesto por cinco procesos de apoyo que son aplicables a la Esace: (1) gestión y educación militar, (2) gestión de ciencia y tecnología, (3) instrucción-entrenamiento y reentrenamiento, (4) ciencias militares y (5) patrimonio. Este macroproceso establece además una guía para desarrollar las actividades que le competen cumpliendo criterios de calidad.

Respecto a los resultados de las evaluaciones derivadas de los planes de mejoramiento, la Esace ha empleado procesos de redimensión curricular, análisis de factores de riesgo de la calidad educativa, potenciación del área investigativa y concursos de mérito con el fin de contar con personal civil idóneo para desarrollar las diversas tareas. Esto es una muestra de la calidad como un proceso de mejora continua.

En este sentido también se debe destacar que el interés por generar una cultura de la investigación ha forjado diferentes prácticas de enseñanza y socialización de los aprendizajes. Un ejemplo de esta transformación es el proceso de gestión de ciencia y tecnología, cuyo objetivo es "desarrollar actividades de investigación para generar ciencia y tecnología dentro de la fuerza; con el fin de dar respuesta a las necesidades del Ejército" (Ministerio de Defensa Nacional, 2018, p. 3). Se trata de un práctica educativa que debe fomentar la cultura de la investigación, el desarrollo tecnológico y la innovación, hasta generar propuestas de proyectos, desarrollo y seguimiento técnico de proyectos, proteger nuevo conocimiento y su aplicación interna y/o externa a la fuerza (Ministerio de Defensa Nacional, 2018, p. 3).

Específicamente, la gestión de ciencia y tecnología está compuesta por diez actividades que parten desde la formulación de directrices hasta el control y seguimiento de cada una de las actividades. Como evidencia de dichas tareas se obtienen diferentes productos: directivas de ciencia y tecnología; producción científica; informes de actividades; radicación y certificado de derecho de autor; licencias para la explotación y uso de los proyectos de investigación realizados; planes de mejoramiento, entre otros.

Estas actividades se formularon a partir de la Directiva Permanente 000153 del 8 de agosto de 2018, donde se estableció la organización y funcionamiento del Sistema de Ciencia, Tecnología e Innovación (Departamento de Educación Militar, 2018). En ese documento se establecieron los lineamientos que buscan promover la investigación científica con el objetivo de solucionar problemáticas de toda índole en la institución. Asimismo, especifica los actores obligados y las disposiciones administrativas que abarcan responsabilidades financieras y de propiedad intelectual. Menciona también que los programas de investigación deben asumir los costos de las investigaciones con el presupuesto que el Ministerio de Defensa 
le asigna. Respecto a la propiedad intelectual, determina que se debe tener control sobre sus derechos.

Otra característica de la directiva es que cuenta con un compilado de anexos donde se identifican los lineamientos de investigación, los procedimientos de divulgación científica, la implementación y la adquisición de tecnologías en los programas de I + D + I, además de que direcciona los formatos establecidos para el sistema de ciencia y tecnología.

\section{Metodología}

El presente estudio forma parte del proyecto de investigación Práctica Pedagógica en Contextos de Formación Militar, el cual hace énfasis en el análisis de diferentes aspectos de los procesos de enseńanza y aprendizaje. Como parte de los resultados de esta investigación se identificaron y analizaron tópicos relacionados con la calidad educativa a través del análisis documental de "periódicos, actas científicas, conclusiones de simposios y seminarios y/o cualquier tipo de publicación considerado como fuente de información" (Martín \& Lafuente, 2017, p. 11). Estos datos fueron recolectados a través de fichas bibliográficas para su posterior análisis.

En el proceso de investigación se hizo la revisión de otros estudios relacionados con la formación en contextos militares con el fin de identificar categorías deductivas. Esta información se recolectó también por medio de la revisión y consulta de diferentes fuentes bibliográficas: Redalyc, Scielo, Scopus, Google Scholar, entre otras, que serán las bases de datos con las cuales contará el Ejército Nacional.

Para analizar esta información, se construyó una matriz de análisis de categorías a partir de una ecuación de búsqueda que arrojó cuáles son los documentos relevantes que aportan al objetivo propuesto. La información se seleccionó con base en una lista significativa de palabras clave que fue construida previamente para analizar los procesos de calidad, específicamente en investigación para la Esace. Estas palabras clave se combinaron mediante comandos y operadores de búsqueda previamente seleccionados con el objetivo de hacer una revisión sistemática a través de la triangulación abierta y axial.

Asimismo, se hizo una revisión sobre la producción científica posterior a la implementación del sistema de educación en la institución con el propósito de analizar la influencia de los estándares de calidad en los procesos de investigación. Se debe señalar que este material se encuentra ubicado en la biblioteca de la institución. Finalmente, se analizaron los resultados para identificar los elementos esenciales de la reflexión. 


\section{Resultados}

El análisis de la información evidencia que el Ejército Nacional ha avanzado significativamente en los procesos de educación. Específicamente, la Esace ha materializado este avance en la calidad de los graduados y en las líneas de investigación que coordinan y guían la producción o enfoque de los proyectos de investigación asignadas por Cedoc a la institución:

1. Sistemas de simulación, generación de entornos virtuales y digitales.

2. Pedagogía de desarrollo humano y socialización.

3. Investigación de operaciones. Teoría de la decisión y doctrina.

Se puede afirmar que estas temáticas se desarrollan en pro de la innovación, ya que se identificó que concuerdan con un periodo y contexto social concreto. Un ejemplo son los proyectos de grado que analizan la funcionalidad de los drones como herramienta tecnológica en diferentes áreas o procesos del Ejército Nacional.

Respecto a la estructura de estos proyectos de investigación, se identificó un cambio en la extensión del documento, la citación y las formas de abordar las temáticas. Anteriormente tendían a ser trabajos extensos y no se controlaba la concordancia entre las citas en el texto y el listado de referencias, pues se encontraron trabajos con citas sin referencia y referencias sin citas, lo cual puede ser un indicador de plagio. En contraste, actualmente esta problemática se controla usando programas que permiten identificar el nivel de coincidencia, los trabajos tienden a ser más cortos, pero puntuales, y se desarrolla la temática de manera completa sin necesidad de extenderse. Además no solamente existe la publicación de monografías, también se puede gestionar el conocimiento mediante la elaboración de ensayos, artículos y capítulos de libro.

Asimismo, se evidenció que anteriormente no se contaba con formatos o algún documento mediante el cual el autor del proyecto cediera los derechos de sus trabajos a la Esace. Por fortuna, esta problemática se solucionó a través del Formato de derechos de autor, en el cual se establece la responsabilidad que tiene la institución sobre esa información. Además, el área de ciencia e investigación de la Esace, en articulación con la biblioteca de la entidad, se encarga de guardar permanentemente los formatos de cesión de derechos de autor, ya que esta es la prueba vital de que la escuela tiene derechos sobre la producción intelectual de los alumnos que completan los programas de posgrado. Estos resultados evidencian el control que ha tenido la Escuela desde que se implementó el sistema de educación, que está en constante seguimiento y por lo tanto facilita el desarrollo de planes de mejoramiento. 
También se encontró que el área de gestión documental cuenta con los folios de vida de cada docente junto con el documento expedido para su contratación, lo cual evidencia la transparencia del proceso académico que lleva el docente seleccionado para las vacantes de investigación —el aula virtual el docente encargado del área académica publica información sobre su trayectoria académica- Al respecto se debe agregar que el perfil del docente que desarrolla el programa de investigación determina las características que de cumplir para desempeñar su cargo, como tener un nivel de educación superior al que va a dictar y una experiencia que evidencie su conocimiento en el campo laboral. Así, actualmente la institución tiene personal docente formado en investigación, la mayoría cuenta con maestría y postulantes a un programa doctoral.

Como se observa, estas características garantizan a los alumnos de la Esace una formación integral acorde con la nueva realidad del país, la cual le permite integrarse con la sociedad y tener una perspectiva de investigación acorde con las necesidades del cambiante ambiente operacional y de la amenaza. En este sentido, las competencias en investigación que desarrolla el egresado se refieren al proceso de búsqueda y generación de conocimiento, tareas que desarrolla mediante una actividad intelectual compleja caracterizada por la creatividad del acto, la innovación de ideas, los métodos rigurosos utilizados y la validación y juicio crítico de pares.

\section{Conclusiones}

En esta reflexión se identificó que la Esace, en articulación con el Ministerio de Educación y la realidad nacional, suministra a los militares en formación herramientas metodológicas para construir sistemas socialmente robustos.

Dando cumplimiento al Plan Estratégico del Sistema Educativo (Pese), la Escuela ha propuesto soluciones o recomendaciones innovadoras, basadas en investigaciones de alto rigor técnico, tecnológico y científico, que involucran la transferencia de conocimiento, necesidades o desafíos en la Fuerza. Esto se evidencia en la producción científica que no solo aborda las problemáticas que hacen deficiente a la institución, sino que además hace propuestas de mejora o identifica conflictos antes no contemplados. Estos trabajos, además, permiten establecer una trazabilidad de las líneas de investigación de la institución y su efectividad en la formación militar.

A pesar de que se emplea un sistema de educación con base en los estándares de calidad definidos por la Unesco, es necesario reconocer que el proceso todavía no se adapta completamente a estos requerimientos. Concretamente, la transición y adaptación del personal y los estudiantes a una nueva exigencia académica implica 
que deben interiorizar nuevas misiones y habilidades, de tal manera que resultan fundamentales cada uno de los planes de mejoramiento que se hacen anualmente en la institución.

Teniendo en cuenta lo anterior, se puede afirmar que este proceso de transformación educativa ha fortalecido las competencias académicas y humanas de los estudiantes, lo cual da valor agregado a la calidad de los programas académicos y constituye una ventaja frente a otras instituciones de educación superior. Así, los egresados de la Esace son capaces de abordar enfoques inter- y transdisciplinares a través de una visión integrada y compleja de la realidad gracias a los elementos educativos y pedagógicos que les ha ofrecido la Escuela, los cuales además les permiten generar nuevas formas de aprender a analizar los problemas emergentes del diario vivir.

\section{Agradecimientos}

Esta investigación ha sido realizada gracias al apoyo de la Escuela de Armas Combinadas del Ejército.

\section{Financiación}

Los recursos humanos y materiales necesarios para desarrollar este capítulo fueron solidarios, ya que fueron proporcionados por la Escuela de Armas Combinadas del Ejército y la Escuela Militar de Cadetes "General José María Córdova”. Los autores declaran que no existe ningún potencial conflicto de interés relacionado con el capítulo.

\section{Referencias}

Asociación Colombiana de Universidades [Ascun]. (2006). Indicadores para la autoevaluación con fines de acreditación institucional. Bogotá, D. C.: Asociación Colombiana de Universidades. Recuperado de https://www.mineducacion.gov.co/CNA/1741/articles-186376_indi_acreinstit.pdf

Buitrago, F. L. (2006). La influencia de los Estados Unidos en el Ejército colombiano 1951-1959. Revista de Estudios Sociales, (25), 123-124.

Centro de Instrucción Militar. (mayo 4 de 2017). Reseña histórica [información en página web]. Recuperado http://cemil.edu.co/index.php/informacion-de-interes/resena-historica/

Consejo Nacional de Acreditación [CNA]. (2013). Lineamientos para la acreditación de programas de pregrado [documento en línea]. Recuperado de https://www.cna.gov.co/1741/articles-186359_ pregrado_2013.pdf 
Departamento de Educación Militar. (agosto 9 de 2018). Directiva Permanente 000153: Organización y Funcionamiento del Sistemas de la Ciencia, Tecnología e Innovación.

Ejército Nacional de Colombia. (s. f.). Reseńa histórica de la Escuela de Caballería [información en página web]. Recuperado de http://escab.cemil.edu.co/index.php/informacion-de-interes/ resena-historical

Ejército Nacional de Colombia. (2017). Proyecto Educativo del Programa Curso Intermedio. Bogotá, D. C.: Ejército Nacional.

Escuela de Armas Combinadas del Ejército [Esace]. (2019a). Programas de cursos militares. Recuperado de: http://esace.cemil.edu.co/index.php/cursos-militares/

Escuela de Armas Combinadas del Ejército [Esace]. (2019b). Programas de posgrado [información en página web]. Recuperado de: http://esace.cemil.edu.co/index.php/programas/posgrados/

Esteban, M. F. (2008). La educación militar en Colombia entre 1886 y 1907. Historia Crítica, (35), 150-175. Recuperado de http://www.scielo.org.co/scielo.php?script=sci_arttext\&pid=S0121-16172008000100009

González, C. S. (2009). La influencia de la participación de Colombia en la guerra de Corea en la construcción de la nueva mentalidad del Ejército Nacional entre 1951 hasta 1982 (Tesis de pregrado). Universidad del Rosario, Facultad de Ciencia Política y Gobierno, Bogotá, D. C., Colombia.

Helg, A. (1986). El desarrollo de la instrucción militar en Colombia en los años 20: estudio del impacto de una misión militar suiza. Revista Colombiana de Educación (17), s. p. https://doi. org/10.17227/01203916.5138

Jaramillo, R. (2004). La calidad de la educación: Hacia un concepto de referencia. Revista de Educación y Pedagogía, 6 (38), 91-100. Recuperado de https://aprendeenlinea.udea.edu.co/revistas/index. php/revistaeyp/article/viewFile/7277/6724

Kalmanovitz, S. (2013). La Constitución de Rionegro. El Espectador. Recuperado de https://www. elespectador.com/opinion/la-constitucion-de-rionegro-columna-449386

Leongómez, E. P. (1987). La profesionalización militar en Colombia (1907-1944). Anal. Politico, (1), 20-39.

Magendzo, A. (1986). Currículum y cultura en América Latina. Santiago de Chile: PIIE.

Martín, S. \& Lafuente, V. (2017). Referencias bibliográficas: indicadores para su evaluación en trabajos científicos. Investigación Bibliotecológica, 31 (71), 151-180. doi: http://dx.doi.org/10.22201/ iibi.0187358xp.2017.71.57814

Melo, L. A., Ramos, J. H. \& Hernández, P. O. (2014). La educación superior en Colombia: Situación actual y análisis de eficiencia. Borradores de Economía, 808. Recuperado de https://www.banrep. gov.co/sites/default/files/publicaciones/archivos/be_808.pdf

Ministerio de Educación Nacional [MEN]. (2015). Decreto único reglamentario del sector educación. Decreto 1075 del 26 de mayo del 2015. Recuperado de https://www.mineducacion.gov.co/ normatividad/1753/w3-article-351080.html

Ministerio de Educación Nacional [MEN]. (2018). Sistema de aseguramiento de la calidad de la educación superior. Decreto 1280 del 25 de julio de 2018. Recuperado de https://www. funcionpublica.gov.co/eva/gestornormativo/norma.php?i=87642

Ministerio de Educación Nacional [MEN]. (2019). Registro calificado de programas académicos de educación superior. Decreto 1330 del 25 de Julio de 2019. Recuperado de: https://es.scribd. com/document/420147596/DECRETO-1330-DEL-25-DE-JULIO-DE-2019-pdf 
Ministerio de Defensa Nacional. (2008). Plan estratégico del Sistemas Educativo de las Fuerzas Armadas 2007-2019 [documento en línea]. Recuperado de https://www.mindefensa.gov.co/irj/ go/km/docs/Mindefensa/Documentos/descargas/estrategia_planeacion/desa_capital/Pagina/ PESE_FINAL.pdf

Organización de las Naciones Unidas para la Educación, la Ciencia y la Cultura [Unesco]. (1996). La educación encierra un tesoro [documento en línea]. Recuperado de http://www.unesco.org/ education/pdf/DELORS_S.PDF

Organización para la Cooperación y el Desarrollo Económicos [Ocde] \& Banco Mundial. (2012). Evaluaciones de políticas nacionales de educación: La educación superior en Colombia. Bogotá, D. C.: OCDE y Banco Mundial.

Páez, C. A., Peón, I. E. \& Ramírez, Y. (2018). Contexto de la seguridad ciudadana en América Latina y el Caribe. Revista Cientifica General José María Córdova, 16 (24), 83-106. https://doi. org/10.21830/19006586.360

República de Colombia. (julio 20 de 1991). Constitución Política de Colombia. Congreso de la Republica. Gaceta Constitucional, n. ${ }^{\circ} 116$. Recuperado de http://www.secretariasenado.gov.co/ senado/basedoc/constitucion_politica_1991.html

República de Colombia. (diciembre 28 de 1992). Ley 30 de diciembre 28 de 1992. Congreso de la República. Diario Oficial, n. ${ }^{\circ}$ 40.700. Recuperado de https://www.cna.gov.co/1741/articles-186370_ley_3092.pdf

Triana, R. E. (junio de 2011). La Escuela Militar de 1880 a 1907: Difícil transición. Revista Científica General José María Córdova, (9), 323. https://doi.org/10.21830/19006586.258 